\title{
CONTROLE ESTATÍSTICO DE PROCESSO PARAMÉTRICO E NÃO PARAMÉTRICO EM UMA INDÚSTRIA DE LATICÍNIOS
}

\section{Parametric and non-parametric Statistical Process Control in a dairy industry}

\begin{abstract}
Luiz Henrique Marra da Silva Ribeiro ${ }^{I *}$, Tatiane Gomes de Araújol, Eric Batista Ferreira ${ }^{I}$, Jovelino Elias Zamboni ${ }^{2}$
\end{abstract}

\section{RESUMO}

O Brasil é um dos maiores produtores mundiais de leite bovino, sendo que grande parte é destinada para fabricação de queijos. Para que a indústria queijeira se mantenha, o leite, sua principal matéria-prima, deve ser de qualidade. Sendo a qualidade do leite avaliada por diversas variáveis em fluxo contínuo, o controle estatístico de processo (CEP) pode ser uma ferramenta para monitorá-la. Neste trabalho foram utilizadas cartas de controle paramétricas e não paramétricas para verificar se o processo de recebimento de leite em uma queijaria da cidade de São Luís de Montes Belos, Goiás, estava sob controle estatístico. Foram verificadas normalidade e simetria dos dados, e como esperado, o CEP paramétrico se mostrou aparentemente mais poderoso. Os autores sugerem a utilização de CEP como ferramenta para assegurar a qualidade de laticínios, mas sempre verificando se as pressuposições de cada metodologia são atendidas. Em específico, se verificada normalidade, as cartas paramétricas são mais poderosas e por isso, são aconselhadas.

Palavras-chave: gestão da qualidade; leite; queijo.

\begin{abstract}
Brazil is one of the world's largest producers of bovine milk, and much of it is destined for cheese production. In order to maintain the cheese industry, the milk, which is its main raw material, must hold a high quality. Given that the milk quality

1 Universidade Federal de Alfenas, Rua Gabriel Monteiro da Silva, 700, centro, 37130-001, Alfenas, MG, Brasil. E-mail: luiz.marra@outlook.com

2 Técnico em Laticínios; jovelino.zamboni@laticiniosuai.com.br

* Autor para correspondência.
\end{abstract}


is assessed by many variables in continuous flow, the statistical process control (SPC) can be one tool to monitor them. In this paper, parametric and non-parametric control charts were used to verify if the milk receiving process in one cheese industry located in the city of São Luís de Montes Belos, in the state of Goiás, Brazil, was under statistical control. Normality and symmetry of the data were verified and, as expected, the parametric SPC was apparently more powerful. The authors suggest the utilization of the SPC as a tool to ensure the quality in the dairy industry, but always verifying if the assumptions of each methodology are met. Specifically, if normality is verified in the data, the parametric charts are more powerful, and they are recommended for this case.

Keywords: quality management; milk; cheese.

\section{INTRODUÇÃO}

O Brasil é o sexto maior produtor mundial de leite bovino, com um aumento anual de produção na faixa de $4 \%$. Cerca de $35 \%$ dessa produção é destinada à produção de queijos, sendo Mussarela, Prato e Frescal os mais prozidos. A produção anual brasileira de queijos é de 488 mil toneladas (DIAMANTINO, 2011). Essa atividade representa uma das atividades mais importantes das que envolvem laticínios (QUEIROGA et al., 2009).

O queijo possui alto valor nutritivo, é indicado pela presença de cálcio e proteínas de alto valor biológico, fósforo, zinco, iodo, selênio, vitaminas, oligoelementos, alto teor de lipídios, e pelas características sensoriais que vêm conquistando o paladar dos consumidores (SILVEIRA JÚNIOR et al., 2012; SERPA et al., 2009; OLIVEIRA et al., 2013). Além disso, estudos mostram que esse queijo possui bons aspectos probióticos e aspectos de segurança, o que indica sua importância para os brasileiros (GOMES et al., 2011).

A procedência do leite interfere na qualidade nutricional e sensorial do queijo (SILVEIRA JÚNIOR et al., 2012). Isso ocorre por vários fatores, sendo o primeiro, que teores de proteína e gordura do leite são algumas características que podem influenciar a composição final do queijo (CICHOSKI et al., 2008). Além disso, quanto maior o extrato seco do leite, ou seja, teores de gordura, açúcar, proteína e sais minerais, mas principalmente gordura e proteína, maior o rendimento do derivado (OLIVEIRA et al., 2013). Essas características são tão importantes que são controladas pela Portaria $n^{\circ} 56$ do Diário Oficial da União $\mathrm{n}^{\circ} 234$, que apresenta que devem ser controlados, dentre outros fatores, teores de gordura, densidade relativa e proteína (QUEIROGA et al., 2009).

Alterações no teor de gordura do leite, por exemplo, podem ocasionar alterações no $\mathrm{pH}$, na umidade e no conteúdo de proteína do queijo fabricado com esse leite (BRITO, 1998). Além do mais, dos fatores que influenciam o teor de gordura do queijo, o teor inicial de gordura do leite é um dos mais importantes (CICHOSKI et al., 2008). Já a gordura do leite pode ser influenciada por fatores genéticos, idade, ambientais e climáticos, e alimentação dos animais, dentre outros fatores (SERPA, et al., 2009; JUSTUS et al., 2011; OLIVEIRA et al., 2013).

A gordura, também contribui para as características sensoriais, mas se consumida em excesso, pode causar doenças coronárias, obesidade e alguns tipos de câncer. (CICHOSKI et al., 2008). Por isso, a importância de se controlar os teores de gordura no leite utilizado para fabricação de queijos, e isso é possível por ações tomadas na seleção e alimentação do rebanho. 
Considerando-se que o processo é contínuo, espera-se que as características que descrevem o processo sejam também contínuas. Então, assumindo distribuições de probabilidade para essas variáveis, podem-se verificar faixas de valores que ocorrerão com baixa probabilidade. Caso sejam observadas as variáveis em tais faixas, considera-se que houve uma alteração no processo (FERREIRA; OLIVEIRA, 2008). A qualidade e características do queijo estão relacionadas com a qualidade e características do leite, que são controladas em fluxo contínuo pela indústria queijeira. Por isso, segue a importância de um controle estatístico de processo (CEP) sob as características do leite para a finalidade de produção de queijos. E como são várias as variáveis que serão controladas, segue a necessidade de um CEP no controle das características do leite utilizado no processo de fabricação de queijo.

Com o objetivo de verificar o CEP como uma ferramenta para auxiliar a verificação da qualidade na indústria de laticínios, e dentre as cartas de Shewhart paramétricas e não paramétricas via teste de postos sinalizados de Wilcoxon, quais melhor se adequam em quais situações foram analisados valores reais de variáveis importantes para o leite quando esse chega à indústria queijeira, e também foram verificados os diferentes efeitos de cartas de controle paramétricas e não paramétricas aos dados.

\section{MATERIAL E MÉTODOS}

Foram analisadas amostras diárias de leite recebido no município de São Luís de Montes Belos, Goiás no ano de 2015. Assim que o leite foi recebido, informações sobre o fornecedor e volume entregue foram computadas e retiradas uma amostra para análises de densidade, teor de gordura e DPC (crioscopia). Além disso, foram analisados o extrato seco total (EST) e extrato seco desengordurado (ESD).
O número de fornecedores e lotes por fornecedores variaram diariamente. Logo, o tamanho amostral (subgrupo racional) também variou diariamente. Neste caso desbalanceado, as cartas de controle devem possuir limite inferior de controle (LIC) e limite superior de controle (LSC) calculados conforme o tamanho amostral de cada dia. Como são cinco as variáveis monitoradas, optou-se por uma abordagem por matrizes do CEP.

Como o fabricante não possuía o $\mathrm{CEP}$ implantado, o processo foi considerado como estando na fase inicial (fase I). Segundo Qiu (2014), é nessa fase que características que representam o processo são verificadas e é checado se essas variáveis estão sob controle estatístico. Caso não estejam, ações devem ser tomadas para corrigir o processo e novas análises devem ser realizadas para verificar se as ações foram suficientes para colocar o processo sob controle estatístico. $\mathrm{Na}$ fase I, cartas de controle de Shewhart são aconselhadas, pois são mais simples de serem feitas e são relativamente capazes de verificar grandes deslocamentos das variáveis (QIU, 2014). Portanto, cartas de controle de Shewhart, em sua abordagem paramétrica e não paramétrica foram adotadas nesse trabalho.

A distribuição normal possui uma grande importância na Estatística, tanto na parte prática quanto teórica. Logo, se uma variável segue distribuição normal, mesmo que aproximadamente, a inferência sobre essa variável é facilmente realizada, e isso não é diferente para cartas de controle (QIU, 2014). No caso de controle de médias, se essas seguem distribuição normal, o que é previsto acontecer pelo teorema central do limite quando o tamanho amostral é grande o suficiente (MAGALHÃES, 2006), cartas de controle via testes paramétricos devem ser preferidas, pois são mais sensíveis que os testes não paramétricos (QIU, 2014).

No entanto, há vários casos em que a normalidade não pode ser assumida para 
certas variáveis. Nestes casos, vários autores informam que testes paramétricos não são confiáveis. Há indícios de que quando uma variável não segue uma distribuição normal e cartas de controle de Shewhart padrão são utilizadas, os intervalos entre paradas fora de controle $\left(\mathrm{ARL}_{0}\right)$ ficam bem menores do que deveriam, o que equivale a dizer que muito esforço é gasto em momentos que o processo está sob controle. Nestes casos, há vários métodos não paramétricos que podem ser utilizados. Caso as variáveis possuam simetria, uma forma de se delimiar LIC e LSC indicado é a soma de postos sinalizados de Wilcoxon (QIU, 2014).

Portanto, desenvolveram-se em operações matriciais limites superiores e inferiores de controle da média e desvio-padrão para cartas de controle Shewhart paramétrico e também limites não paramétricos, ambos sensíveis ao tamanho amostral, sendo o primeiro indicado para variáveis que apresentem normalidade ou normalidade aproximada e o segundo para quando a distribuição da variável não foi verificada.

Pelas dificuldades encontradas pelos autores na localização das fórmulas matriciais para os limites de controle por matrizes, elas serão apresentadas abaixo para fins de divulgação científica.

\section{Controle Estatístico de Processos}

\section{Cartas paramétricas}

O CEP de Shewhart considera que a média e a variância das variáveis seguem distribuições normais, e que elas são independentes. Já para o caso múltiplo, seja $X_{i}$ a matriz de dados observados no dia $i$, com $p$ variáveis nas colunas e $n_{i}$ observações nas linhas e 1 um vetor composto por $n$ uns. Assim, sendo $\hat{\mu}_{i}$ o vetor de médias e $\hat{\sigma}_{i}^{2}$ o vetor de variâncias do dia $i$ :

$$
\hat{\mu}_{i}=X_{i}^{t} 1\left(\frac{1}{n_{i}}\right)
$$

onde $\hat{\mu}_{i}$ é o vetor de médias do dia $i$. Já para os vetores de variâncias e desvio-padrão $\hat{\sigma}_{i}^{2}$ e $\hat{\sigma}_{i}:$ :

$$
\begin{gathered}
\hat{\sigma}_{i}^{2}=\operatorname{diag}\left(\frac{1}{\left(n_{i}-1\right)}\left[X_{i}^{t} X_{i}-\left(\frac{1}{n_{i}}\right) X_{i}^{t} 11^{t} X_{i}\right]\right) ; \\
\hat{\sigma}_{i}=\sqrt{\hat{\sigma}_{i}^{2}}
\end{gathered}
$$

Logo, a matriz de desvios-padrão $M \hat{\sigma}$ e o vetor de médias dos desvios-padrão $\hat{\sigma}_{i_{\text {med }}}$ são dados por:

$M \hat{\sigma}=\left[\hat{\sigma}_{1}, \hat{\sigma}_{2}, \ldots, \hat{\sigma}_{n}\right] ; \quad \hat{\sigma}_{m e d}=M \hat{\sigma} 1\left(\frac{1}{n_{i}}\right)$

Dessa forma, depois de criado o vetor de constantes $d 3(n)$ e $n_{i}$ :

$$
\begin{gathered}
d 3(n)=\left[d 3\left(n_{1}\right), d 3\left(n_{2}\right), \ldots, d 3\left(n_{n}\right)\right] \\
n_{i}=\left[n_{1}, n_{2}, \ldots n_{n}\right],
\end{gathered}
$$

para que a matriz de limites inferiores de controle para média $\left(L I C_{\text {med }}\right)$ e limites superiores de controle para a média $\left(L S C_{\text {med }}\right)$ possam ser verificadas da seguinte forma:

$$
\begin{aligned}
& \left(L I C_{\text {med }}\right)=\hat{\mu}_{i}+\hat{\sigma}_{\text {med }} \otimes\left[\frac{\mathrm{Z}_{\alpha / 2}}{d_{3}(n) \sqrt{n_{i}}} 1\right]^{t} ; \\
& \left(L S C_{\text {med }}\right)=\hat{\mu}_{i}-\hat{\sigma}_{\text {med }} \otimes\left[\frac{\mathrm{Z}_{\alpha / 2}}{d_{3}(n) \sqrt{n_{i}}} 1\right]^{t},
\end{aligned}
$$

além disso, se tem o vetor para as linhas centrais das cartas de médias dado por $\hat{\mu}_{i}$. 
Já para os limites inferior e superior de controle dos desvios-padrão, respectivamente $\left(L I C_{D P}\right)$ e $\left(L S C_{D P}\right)$, são encontrados da seguinte forma matricial:

$$
\begin{gathered}
L I C_{D P}=\hat{\sigma}_{\text {med }}-\hat{\sigma}_{\text {med }} \mathrm{Z}_{\alpha / 2} \otimes 1 \frac{\sqrt{1-d_{3}(n)}}{d_{3}(n)}, \\
\left(L S C_{\text {med }}\right)=\hat{\mu}_{i}-\hat{\sigma}_{\text {med }} \otimes\left[\frac{\mathrm{Z}_{\alpha / 2}}{d_{3}(n) \sqrt{n_{i}}} 1\right]^{t}
\end{gathered}
$$

Então, para as linhas centrais das cartas de controle para a variância, utiliza-se o vetor de variâncias médias $\hat{\sigma}_{\text {med }}$, e para os limites inferiores e superiores, considerando-se os tamanhos amostrais, tem-se as matrizes $L I C_{D P}$ e $L S C_{D P}$, respectivamente.

Desta forma, os pontos dos gráficos representam os valores médios e variâncias dos dados. A reta central representa a média da média e da variância. As probabilidades de ocorrerem valores acima do limite superior e abaixo do limite inferior das cartas são baixas. Então, sendo os dados independentes, uma tendência qualquer pode indicar alteração do sistema, assim como pontos fora dos limites de controle.

\section{Cartas não paramétricas}

Segundo Qiu (2014), as cartas de controle de Shewhart não paramétricas se mostraram menos sensíveis que as cartas paramétricas. Isso geralmente é acentuado em duas ocasiões: a primeira, porque cartas paramétricas são mais poderosas quando há normalidade nas variáveis, mesmo que aproximadamente. E o segundo, porque para a utilização de postos sinalizados de Wilcoxon, necessita-se ter variáveis simétricas, coisa que geralmente falta aos dados.

Ainda segundo o autor, o teste de postos sinalizados de Wilcoxon pode ser utilizado para gerar limites de controle para variáveis da seguinte forma, sendo $X_{m d(i)}$ a mediana dos valores observados do tempo $i$ :

Passo 1: Ordena-se de forma crescente os dados, considerando-se $\left|X_{i}-X_{m d(i)}\right|$ e cria-se postos, sendo o menor valor " 1 " e o maior " $n$ ". Caso haja valores repetidos de $\left|X_{i}-X_{m d(i)}\right|$, utiliza-se a média dos postos para todos elementos.

Passo 2: Define-se $S_{+}$, a soma dos postos de todos elementos que possuírem valor $\left(X_{i}-X_{m d(i)}\right)$ não negativos.

Passo 3: compara-se os valores observados de $S_{+}$com os limites de controle.

Em casos onde $n$ é grande o suficiente $(n>20)$, a distribuição de $S_{+}$é aproximadamente normal com média e variância, respectivamente: $\hat{\mu}_{\mathrm{S}_{+}}=n_{i}\left(n_{i}+1\right) / 4 \mathrm{e}$ $\hat{\sigma}_{S_{+}}^{2}=n_{i}\left(n_{i}+1\right)\left(2 n_{i}+1\right) / 24$, logo, seguem as fórmulas dos limites de controle univariados:

$$
L I C_{S_{+}}=\frac{n_{i}\left(n_{i}+1\right)}{4}-\frac{n_{i}\left(n_{i}+1\right)}{4} \frac{\mathrm{Z}_{\alpha / 2}}{d_{3}\left(n_{i}\right) \sqrt{n_{i}}}
$$

$$
\sqrt{n_{i}\left(n_{i}+1\right)\left(2 n_{i}+1\right) / 24}
$$

$$
\begin{gathered}
L C_{S_{+}}=\frac{n_{i}\left(n_{i}+1\right)}{4} ; \\
L S C_{S_{+}}=\frac{n_{i}\left(n_{i}+1\right)}{4}+\frac{n_{i}\left(n_{i}+1\right)}{4} \frac{\mathrm{Z}_{\alpha / 2}}{d_{3}\left(n_{i}\right) \sqrt{n_{i}}} \\
\sqrt{n_{i}\left(n_{i}+1\right)\left(2 n_{i}+1\right) / 24}
\end{gathered}
$$


Como este tipo de carta de controle considera apenas o número de observações por período, não é necessário um método matricial de estimação, pois para todas as $p$ variáveis que verificaremos possuem o mesmo número de observações para $n$ amostras. Assim, para o modelo não paramétrico, apenas uma carta de controle é criada, e todas as somas sinalizadas das $p$ variáveis são plotadas nessa carta.

Todas as operações matriciais e os gráficos foram realizados com o auxílio do software estatístico R (CORE TEAM, 2018).

\section{RESULTADOS E DISCUSSÃO}

Foram realizados testes de normalidade para as médias, considerando o nível de significância de $5 \%$, representados na Tabela 1. Foi verificado que, para algumas variáveis e para alguns períodos, os dados apresentaram valor-p acima do nível de significância considerado, o que é um indício de normalidade, já era esperado pelo teorema central do limite, sendo o tamanho amostral médio das amostrar ser aproximadamente 1.000 .

O coeficiente de assimetria pode ser utilizado para verificar se uma série de dados é simétrica. Na Tabela 2, estão valores absolutos do coeficiente de assimetria, em que valores próximos de zero são sinais de que os dados vêm de uma distribuição simétrica. A pressuposição de simetria não foi atendida por completo. Pode-se verificar que para todas as variáveis, pelo menos um período apresentou valor absoluto do coeficiente de assimetria maiorque1, sendo que ESD apresentou um valor absoluto de 8,8668 .

Tabela 1 - Valores-p para os testes de Shapiro-Wilk para as médias

\begin{tabular}{cccccc}
\hline & Gordura & Densidade & DPC & EST & ESD \\
\hline Valor-p & 0,191 & 0,934 & 0,299 & 0,113 & 0,964 \\
\hline
\end{tabular}

Tabela 2 - Valores do coeficiente de assimetria para as variáveis e dias avaliados

\begin{tabular}{ccccccc}
\hline Amostra & $\begin{array}{c}\text { Tamanho } \\
\text { Amostral }\end{array}$ & Gordura & Densidade & DPC & EST & ESD \\
\hline 1 & 123 & 0,1650 & 0,1180 & 0,1180 & 0,0686 & 0,0039 \\
2 & 113 & 0,1130 & 0,1380 & 0,2400 & 0,0358 & 2,5150 \\
3 & 129 & 0,0585 & 0,0348 & 0,0917 & 0,0119 & 2,2470 \\
4 & 92 & 0,3111 & 0,1482 & 0,1961 & 0,1623 & 0,0892 \\
5 & 116 & 0,1442 & 0,0573 & 0,1454 & 0,0467 & 5,7973 \\
6 & 8 & 0,9937 & 0,1572 & 0,1459 & 0,5368 & 0,4546 \\
7 & 276 & 0,6974 & 0,0895 & 0,0964 & 0,0577 & 0,4044 \\
8 & 138 & 0,0222 & 1,4945 & 2,6917 & 2,4239 & 8,8668 \\
9 & 82 & 1,0221 & 2,1171 & 1,1235 & 0,4617 & 3,3970 \\
10 & 72 & 0,5303 & 0,2062 & 0,8594 & 0,4233 & 0,0040 \\
11 & 81 & 0,0554 & 0,1107 & 0,2931 & 0,2736 & 2,3415 \\
\hline
\end{tabular}


A Figura 1 apresenta as cartas de controle das médias e desvios-padrão das variáveis de interesse.

Pode-se verificar na Figura 1, que apa-
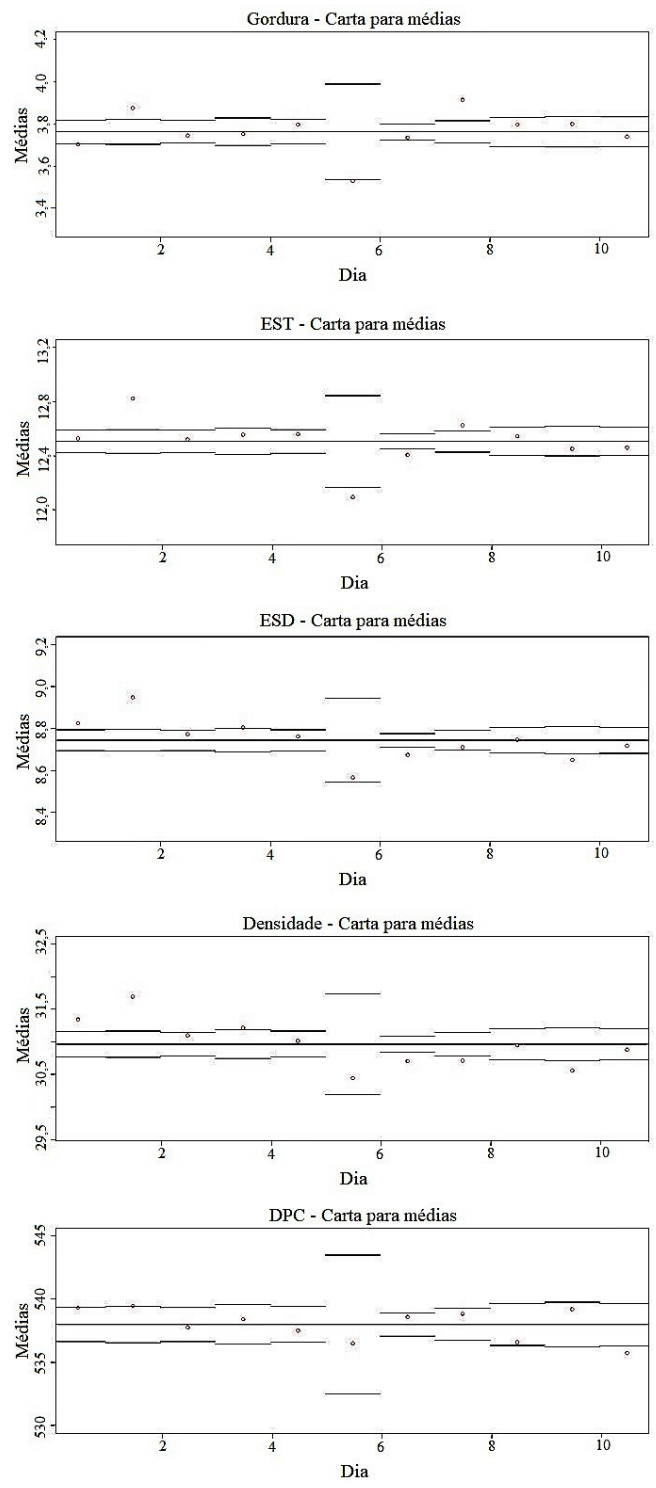

rentemente, todas as variáveis controladas se apresentam fora de controle estatístico. Neste caso, devem-se tomar ações para que o processo entre em controle estatístico (QIU,
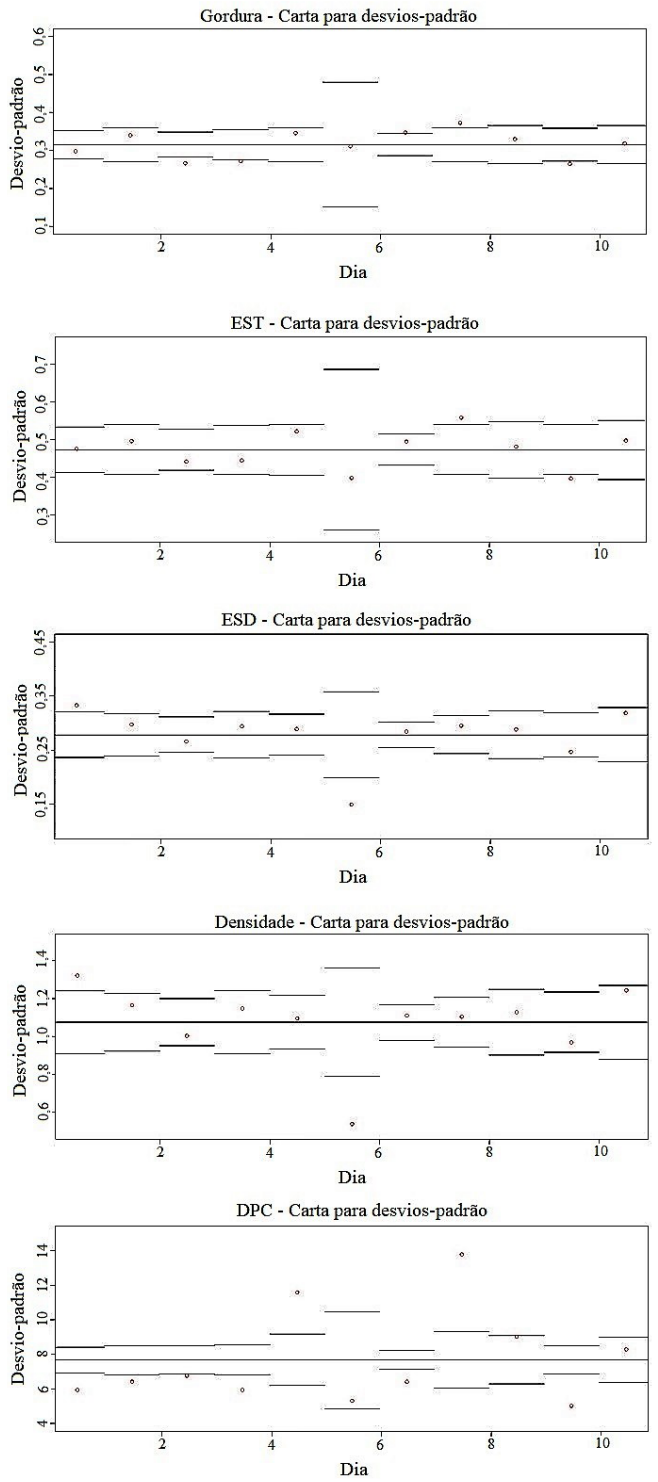

Figura 1 - Cartas de controle paramétricas de médias e desvios-padrão das variáveis de interesse: gordura, EST, ESD, densidade e DPC para os dias avaliados 
2014; FERREIRA et al., 2011). E esse tipo de constatação é de difícil verificação sem a utilização do CEP.

A carta para o teor de gordura apresentou valores fora dos limites de controle em cinco dias, para pelo menos uma carta, para média ou variância. Essas alterações podem implicar em alterações no $\mathrm{pH}$, umidade e taxa proteica do leite (BRITO, 1998), podendo prejudicar a composição do queijo (CICHOSKI et al., 2008). Diante desses fatos, o produtor de queijo pode tomar decisões, em conjunto com o fornecedor de leite, para o controle dessa variável controlando os fatores: genéticos, idade do gado, ambientais e climáticos, e alimentação dos animais, dentre outros fatores (SERPA, et al., 2009; JUSTUS et al., 2011; OLIVEIRA et al., 2013).

Os fatores EST e ESD também apresentaram cinco pontos fora do controle da média ou da variância. Esses indicadores possuem relação com teores de gordura, açúcar, proteína e sais minerais, possuindo relação com o rendimento do queijo (OLIVEIRA et al., 2013). As ações relacionadas com esses fatores, que podem ser ajustadas para um controle das variáveis são: genéticos, idades dos animais do rebanho, produção de leite diária e condições do parto (GALVÃO JÚNIOR, 2010).
A carta de controle para a densidade apresentou pontos fora dos limites de controle para seis dias, considerando-se as cartas para média e variância. Além de possuir relação com EST, ESD e com o teor de gordura, a densidade do leite pode ser utilizada para verificação de fraudes causadas pelo fornecedor (ABRANTES et al., 2014). Assim, o produtor pode utilizar das cartas de controle dessa variável para se proteger de possíveis fraudes no leite, como a adição de água para um maior volume.

A carta de controle para o DPC apresentou nove pontos fora dos limites para cartas de controle da média ou da variância. O DPC está relacionado com o rendimento do leite, e tipo de ordenha, alimentação, idade, dentre outros fatores, podem ser controlados para um melhor controle no DPC (VIEIRA et al., 2010).

A Figura 2 apresenta a carta de controle pelo método não paramétrico de soma de postos de Wilcoxon.

Pode-se verificar pela Figura 2 que, pelas cartas de controle não paramétricas, todas as variáveis estão dentro dos limites de controle e apresentam aparente independência. Isso é explicado por Qiu (2014), que explica que esse método não paramétrico é menos poderoso do que métodos paramétricos, como

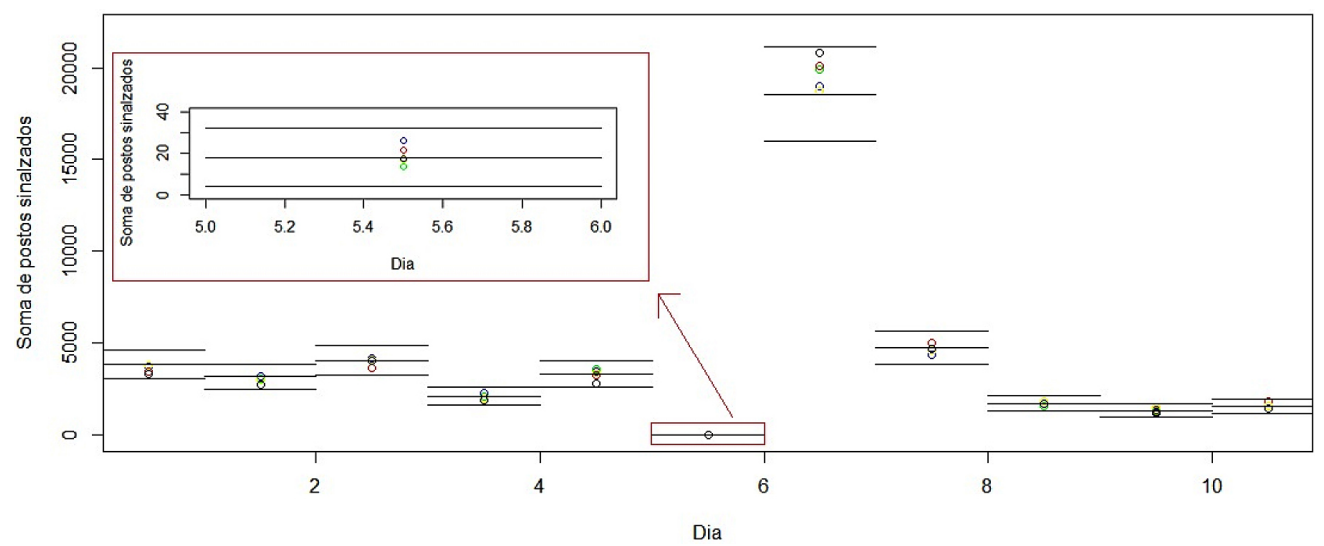

Figura 2 - Cartas de controle pelo método não paramétrico de soma de postos de Wilcoxon 
o caso das cartas de Shewhart. Portanto, segundo essa carta de controle, todas as variáveis consideradas estão em controle estatístico no período avaliado.

\section{CONCLUSÕES}

Conclui-se com esse trabalho, que o controle estatístico de processo pode indicar aos fabricantes de queijo se os fornecedores de leite estão fornecendo leite com variáveis em estado de controle estatístico, e caso não esteja, tomar ações juntamente aos fornecedores, para fazer com que as variáveis que descrevem o leite estejam em controle estatístico.

Aqui foram utilizadas apenas cinco variáveis, mas outras, como acidez, contagem microbiana, e teor de proteína também podem ser verificadas, que como o método sugerido é múltiplo, o acréscimo de variáveis não é tido como um problema.

Os autores sugerem a aplicação de cartas de controle como ferramenta de controle da qualidade do leite, sendo que se verificada a normalidade, a utilização de testes paramétricos deve ser preferida, pois o teste é mais poderoso que o não paramétrico nessa situação. Porém, se não for verificada a normalidade, as cartas de controle não paramétricas podem ser utilizadas, e a soma de postos sinalizados de Wilcoxon pode ser facilmente aplicada, caso haja simetria nos dados.

\section{AGRADECIMENTOS}

O presente trabalho foi realizado com apoio da Coordenação de Aperfeiçoamento de Pessoal de Nível Superior - Brasil (CAPES) - Código de Financiamento 001. Os autores agradecem também à FAPEMIG, CNPq e UNIFAL pelo apoio financeiro.

\section{REFERÊNCIAS}

ABRANTES, M. R.; CAMPÊLO, C. S.; SILVA, J. B. A. Fraude em leite: Métodos de detecção e implicações para o consumidor. Revista do Instituto Adolfo Lutz, v. 73, n. 3, p. 244-251, 2014.

BRITO, J. R. F.; DIAS, J. C. A qualidade do leite. Juiz de Fora: EMBRAPA-CNPGL/São Paulo: Tortuga, 1998. 88p.

CICHOSKI, A. J. et al. Efeito da adição de probióticos sobre as características de queijo prato com reduzido teor de gordura fabricado com fibras e lactato de potássio. Ciência e Tecnologia de Alimentos, v. 28, n. 1, p. 214-219, 2008.

DIAMANTINO, I. M. Efeito de substitutos de gordura na qualidade de queijo Prato com reduzido teor de gordura. 2011. 68 f. Dissertação (Mestrado em Engenharia e Ciência de Alimentos) - Universidade Estadual Paulista, São José do Rio Preto, 2011.

FERREIRA, E. B.; OLIVEIRA, M. S. Controle estatístico da qualidade, UFLA/ FAEPE, 2008.

FERREIRA, E. B. et al. Controle Estatístico de Processo no Resfriamento de Aves: Um Estudo de Caso. Revista da Universidade Vale do Rio Verde, v. 9, n. 2, p. 119-128, 2011.

GALVÃO JÚNIOR, J. G. B. et al. Efeito da produção diária e da ordem de parto na composição físico-química do leite de vacas de raças zebuínas. Acta Veterinaria Brasilica, v. 4, n. 1, p. 25-30, 2010.

GOMES, A. P. et al. Manufacture of lowsodium Minas fresh cheese: effect of the partial replacement of sodium chloride 
with potassium chloride. Journal of Dairy Science, v. 94, n. 6, p. 2701-2706, 2011.

JUSTUS, A. et al. Caracterização física e química de queijos parmesão ralado comercializados na Região Sul de Minas Gerais. Revista do Instituto de Laticínios Cândido Tostes, v. 66, n. 379, p. 16-24, 2011.

MAGAlHÃeS, M. N. Probabilidade e variáveis aleatórias. São Paulo: Edusp, 2006. 411p.

OLIVEIRA, D. F. et al. Caracterização físico-química de queijos Minas Artesanal produzidos em diferentes microrregiões de Minas Gerais. Oikos: Revista Brasileira de Economia Doméstica, v. 24, n. 2, p. 185196, 2013.

QUEIROGA, E. R. C. R. et al. Elaboração ção e caracterização físico-química, microbiológica e sensorial de queijo "tipo minas frescal" de leite de cabra condimentado. Revista Ciência Agronômica, v. 40, n. 3, p. 363-372, 2009.
QIU, P. Introduction to Statistical Process Control. Boca Raton, Florida: CRC, 2014.

R, CORE TEAM. A Language and Environment for Statistical Computing. Vienna, Áustria, 2018. Disponíevl em: <https://www. R-project.org/>. Acesso em 13 set. 2018.

SERPA, L. et al. Destino ambientalmente correto a rejeitos de queijaria e análise de viabilidade econômica. In: INTERNACIONAL WORKSHOP - Advances in Cleaner Production, 2., 2009, São Paulo, SP. Resumos... São Paulo: IW-ACP, 2009. p. 1-10.

SILVEIRA JÚNIOR, J. F. et al. Caracterização físico-química de queijos Coloniais produzidos em diferentes épocas do ano. Revista do Instituto de Laticínios Cândido Tostes, v. 67, n. 386, p. 67-80, 2012.

VIEIRA, V. M. C. et al. Qualidade do leite de vacas coletado em ordenha mecânica e manual em propriedades na região de Aramina-SP. Nucleus Animalium, v. 2, n. 2, p. 21-26, 2010. 